\title{
EVALUATION OF COST-SAVING MACHINE LEARNING METHODS FOR PATIENT BLOOD MANAGEMENT
}

\author{
Davide Brinati ${ }^{1}$, Andrea Seveso ${ }^{1}$, Paolo Perazzo ${ }^{2}$, Giuseppe Banfii and Federico Cabitza ${ }^{3}$ \\ ${ }^{I}$ Dipartimento di Informatica, Sistemistica e Comunicazione, Università degli Studi di Milano-Bicocca \\ Viale Sarca 336, 20126, Milano, Italy \\ ${ }^{2}$ Istituto Ortopedico Galeazzi, Via Riccardo Galeazzi, 4, 20161 Milano, Italy \\ ${ }^{3}$ Dipartimento di Informatica, Sistemistica e Comunicazione, Università degli Studi di Milano-Bicocca \\ Viale Sarca 336, 20126, Milano, Italy
}

\begin{abstract}
Our objective is the development of cost-saving methods for the patient blood management in Galeazzi Orthopedic Institute, a large Italian hospital. The methods have been developed in relation to the known costs of the hospital, both in terms of unused blood bags and drugs. Observational data about 4593 patients have been retrieved, with anagraphical and pre-operational clinical features. Model's performances have been compared to an existing baseline in terms of both accuracy measures (F1, recall, AUC) and saved costs per patient. The proposed methods recorded an enhancement of performances for the adopted measures, demonstrating a possible useful application of machine-learning-based methods for the patient blood management task.
\end{abstract}

\section{KEYWORDS}

Patient Blood Management, Machine Learning, Sensitivity Analysis, Operation Costs

\section{INTRODUCTION}

Blood transfusion is a widely used procedure in hospitalized patients, used in many different clinical conditions, such as traumas, infections, hemorrhages. Notwithstanding this therapy represents a lifesaving treatment for some patients, it is also one of the most overused procedures in hospitals. Unnecessary transfusions often lead to increased risks and costs, due to the suboptimal allocation of such an important good. An efficient strategy aims to improve the transfusion practice by avoiding unhandy operations for the patient and saving blood costs. This is an effective method of promoting high-value cares. Some of these costs could be saved by treating the patient with medicines that reduce the risk of transfusion, thus reducing the costs, since medicines have a lower price than blood bags.

In addition to the economic aspect, a successful patient blood management system diminishes unnecessary allocations of blood bags, which remain available for the patients that actually need transfusions.

Some previous works (Sadana et al. 2000, Hayn, 2017) focused their attention on the optimization of blood transfusion. The latter describes how predictive modelling and machine learning tools applied on pre-operative data can be used to predict the amount of red blood cells to be transfused during surgery and to prospectively optimize blood ordering schedules.

This work aims for evaluating the current patient blood management system of the Italian hospital Galeazzi Orthopedic Institute (Istituto Ortopedico Galeazzi - IOG), a center for medical research in the orthopedic field, based in Milan, and investigate if and how it can be improved, in terms of saved costs. Furthermore, a machine learning method will be proposed and compared with the previous methods.

IOG already has a method to deal with the patient blood management, based on a heuristic approach dictated by clinical expertise. Patients hemoglobin levels are evaluated, and a threshold is set: if the patient presents a hemoglobin value less than 12.9 , then he is likely to undergo a transfusion, and so he is given a set of erythropoietin-stimulating agents in order to decrease the transfusion risks. 


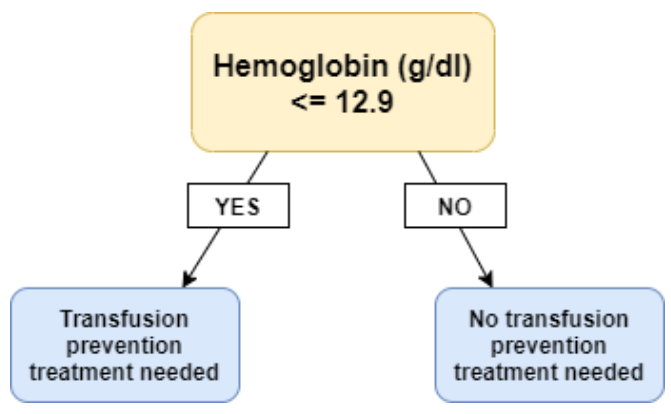

Figure 1. Current strategy at IOG: if the patient hemoglobin level falls below 12.9, transfusion prevention treatment is deemed required, meaning the patient will receive erythropoietin-stimulating drugs

This method seems preventive because it is overly cautious, causing the rise of false positive patients, e.g. those would not require a transfusion, e.g. the drugs administered would be an unnecessary cost. This situation can be visualized in figure 2 .

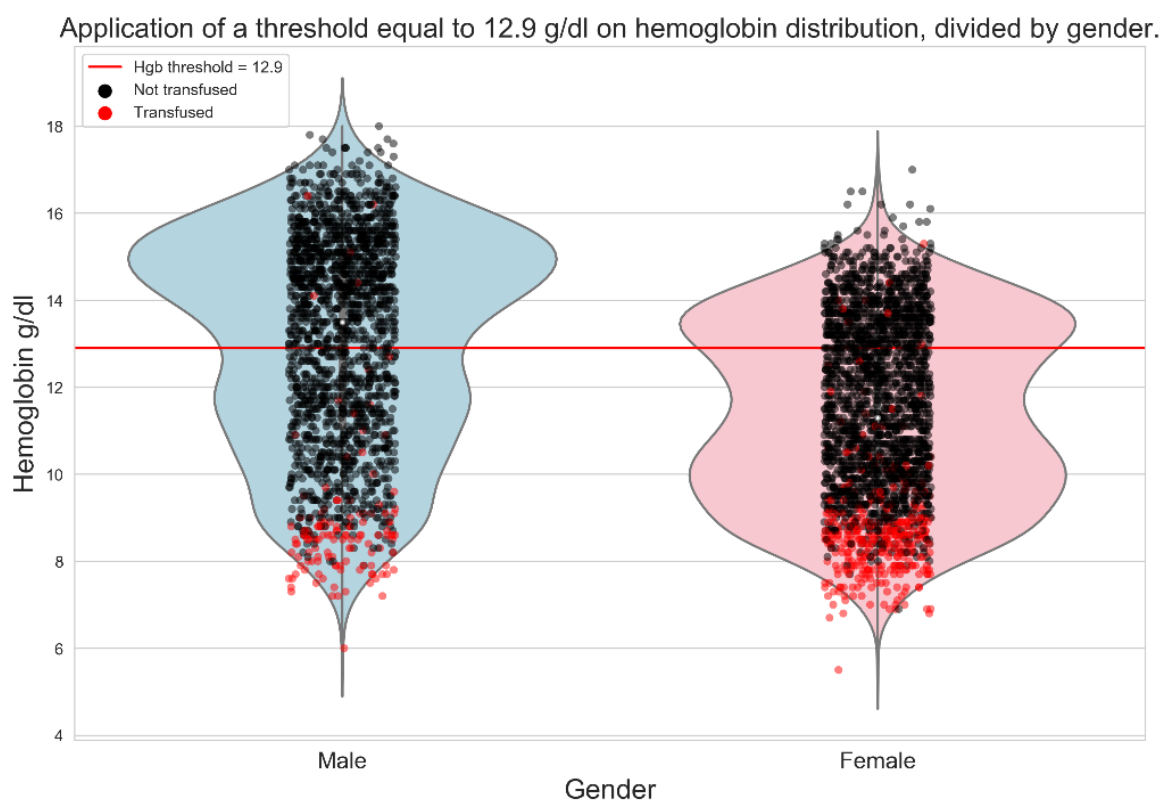

Figure 2. Violin plots showing the distribution of hemoglobin, divided by gender. The red horizontal line denotes the current preemptively method: observations below this threshold are predicted as transfusion requiring and those above as non-transfusion requiring. Points marked in red are patients who have been transfused, while black points are patients who have not received a transfusion. A large portion of patients are classified by the simple existing method as likely to be transfused, even if they will not require it

Our research activity has two goals: the first one concerns if an improvement over the heuristic method based on hemoglobin thresholds is possible by performing a sensitivity analysis on the threshold, using a data-driven approach, also keeping the method as simple as possible. Secondly, we propose a blood-transfusion machine learning classification system to decrease the costs attributable to blood bags. 


\section{METHODS}

\subsection{Dataset Description}

The observational data was made available for this study by Galeazzi Orthopedic Institute, reporting the medical information and anagraphical personal data about 11814 hospitalizations (4593 patients) from January 2019 to May 2019, in particular: patient's age, sex, hemoglobin levels (g/dl), International Classification of Diseases (ICD9) code representing the patient's disorder, the urgency of the operation and the information about whether the transfusion occurred or not. An extended description of the features used for modelling is shown in table 1.

Table 1. Features of the dataset provided by IOG

\begin{tabular}{lll}
\hline Features & Description & Data Type \\
\hline Age & Age at operation date & Numerical \\
Sex & Patient gender & Categorical \\
Hemoglobin $($ HGB $)$ & HGB $(\mathrm{g} / \mathrm{dL})$ & Numerical \\
ICD9 & Operation code & Categorical \\
Urgency & Urgent operation & Categorical \\
Transfused & Transfusion received & Categorical \\
\hline
\end{tabular}
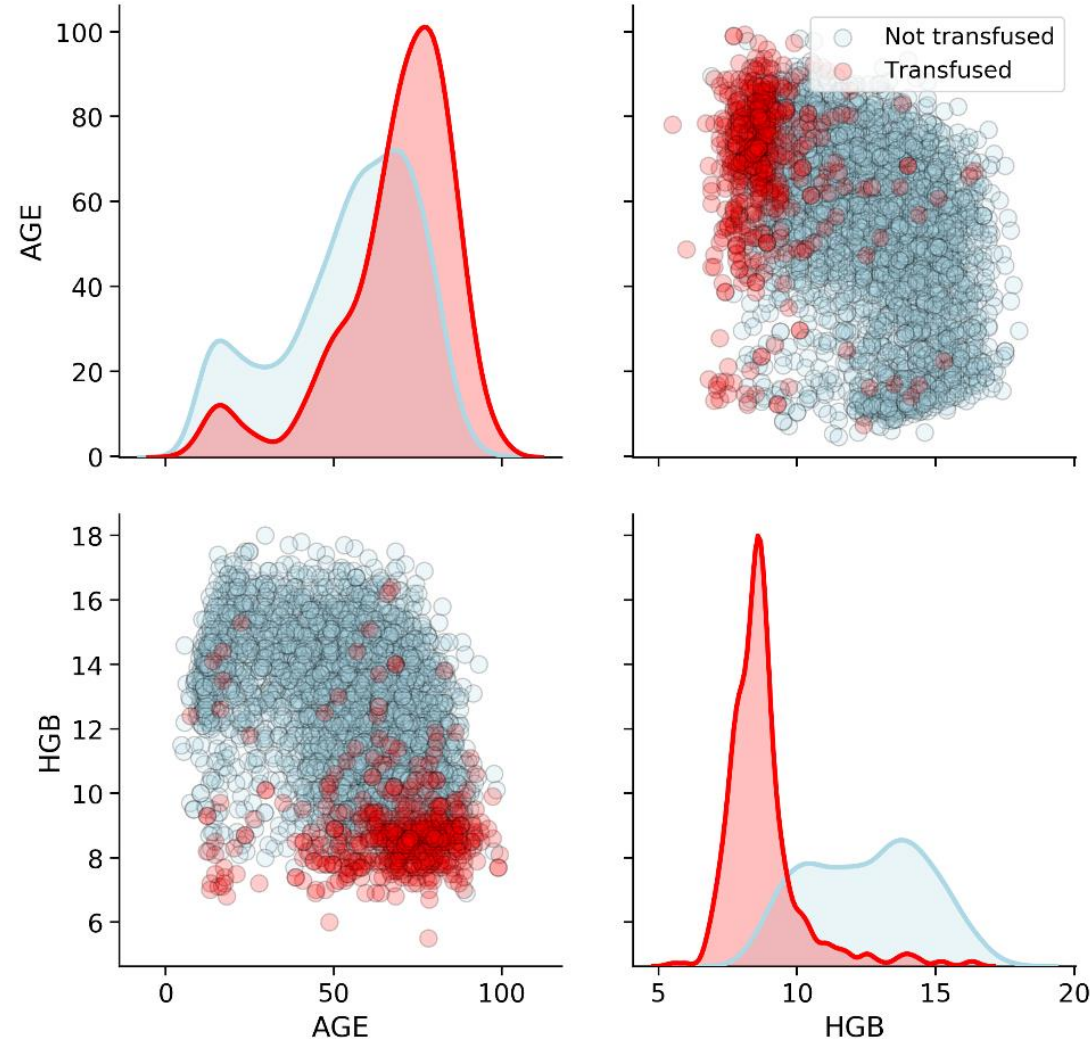

Figure 3. Visualization of the distribution of age and HGB levels in IOG's patients. Color of traces indicates whether the patient was transfused or not, as indicated in the figure's legend

Figure 3 shows the pairwise distribution plots and scatterplots of "Age" and " $H G B$ " variables. Plots denote a negative correlation between " $A g e$ " and " $H G B$ " (Pearson correlation: $\mathrm{r}=-0.43$, p-value $<0.001$ ). In addiction, transfused patients show an " $H G B$ " distribution with lower mean and variance than non-transfused patients. 
The outcome of our analysis is the prediction of whether a hospitalization will require a transfusion (or more) or not during the surgery.

\subsection{Data Manipulation}

The dataset features several samples with the hemoglobin values detected after the transfusion $(n=7530,64 \%)$. This information was made available by the metadata coming with the case-study dataset, which reports the date of surgery and the date of the hemoglobin value detection. To avoid a biased model training, those samples were removed since this work aims to predict possible transfusion, based only on preoperative data.

Missing data was imputed using K-Nearest Neighbor (KNN) imputation (Zhang, 2012), with K=1, meaning the imputation value is taken from the closest patient. The resulting dataset presents 4284 hospitalizations. The categorical variable ICD9, as it contains multiple categories, has been represented using one-hot encoding (Ramaravind et al. 2020).

The target feature, Transfused, presents a class imbalance, with 499 positive cases (11.6\%) and 3785 negative cases $(88.4 \%)$. To deal with this issue, the division of the dataset into training and validation sets was performed in a stratified manner and a SMOTE (Chawla et al., 2002) was applied in order to balance the classes. Smote is a type of data augmentation, which consists generating new minority class instances conditioned on the distribution of known instances.

\subsection{Sensitivity Analysis}

Firstly, we would like to perform a sensitivity analysis in order to optimize the existing method, and to find an optimal threshold that decreases the operation costs. After an analysis performed at IOG, we found that the cost of a False Negative, meaning a patient is classified as not requiring a transfusion when in reality he will, is about six times the cost of a False Positive (FP), meaning when a patient is given the drugs in order to decrease the transfusion risk but he really does not require it. We show the potential cost of misclassification of each possible HGB threshold, and then choose the best overall threshold in terms of cost savings.

\subsection{Machine Learning Classifier}

In order to create the classifier model, we tested a set of machine learning techniques:

- $\quad$ heuristic approaches, e.g. Decision Tree (Safavian, 1991), Random Forest (Kam, 1995) and K-nearest neighbors (Altman, 1992)

- $\quad$ Regression based approach, e.g. Logistic regression (Hosmer et al 2013),

- $\quad$ Separative approach, e.g. Support vector machine (Schölkopf et al. 2002)

10-fold Nested cross-validation has been used to provide an unbiased estimation of model performances. In a nested cross-validation, an inner loop chooses the best set of hyperparameters (chosen from the most common ones used for each model) using a set grid search, and the outer loop evaluates the model performance.

\subsection{Evaluation of Methods}

Due to the imbalance of the Transfused target feature, the evaluation metrics were chosen as the precision, recall, $f$-score, which is the harmonic mean of precision and recall, and Area Under the Curve (AUC). However, what is most important to us (after, of course, patient's health), is the overall decrease in costs in comparison to the existing baseline. This entails, since the cost of FN is six-fold of FP, that in terms of classical evaluation measures the recall is the most important amongst those. 


\section{RESULTS}

\subsection{Sensitivity Analysis Results}

We have performed the sensitivity analysis as described in the "Methods" section, and the results are shown in Figure 4, which visualizes the behavior of the adopted metrics by varying the hemoglobin threshold. The normalized cost is also reported, representing the cost in the $[0,1]$ interval, in order to provide a better graphical comparison with the other performance measures considered. The costs normalization is given by:

$$
\begin{gathered}
\operatorname{cost}_{\text {std }}=\left(\operatorname{cost}-\operatorname{cost}_{\text {min }}\right) /\left(\operatorname{cost}_{\text {max }}-\operatorname{cost}_{\text {min }}\right), \\
\operatorname{cost}_{\text {normalized }}=\operatorname{cost}_{\text {std }} *\left(\operatorname{cost}_{\text {max }}-\operatorname{cost}_{\text {min }}\right)+\operatorname{cost}_{\text {min }} .
\end{gathered}
$$

We can see that for each HGB threshold, the various metrics change correspondingly. In particular, the threshold that minimizes the potential costs is $9.4 \mathrm{~g} / \mathrm{Dl}$, and for this threshold the precision is 0.56 and the recall is 0.86 . The overall cost for each patient is equal to 18.43 euros.

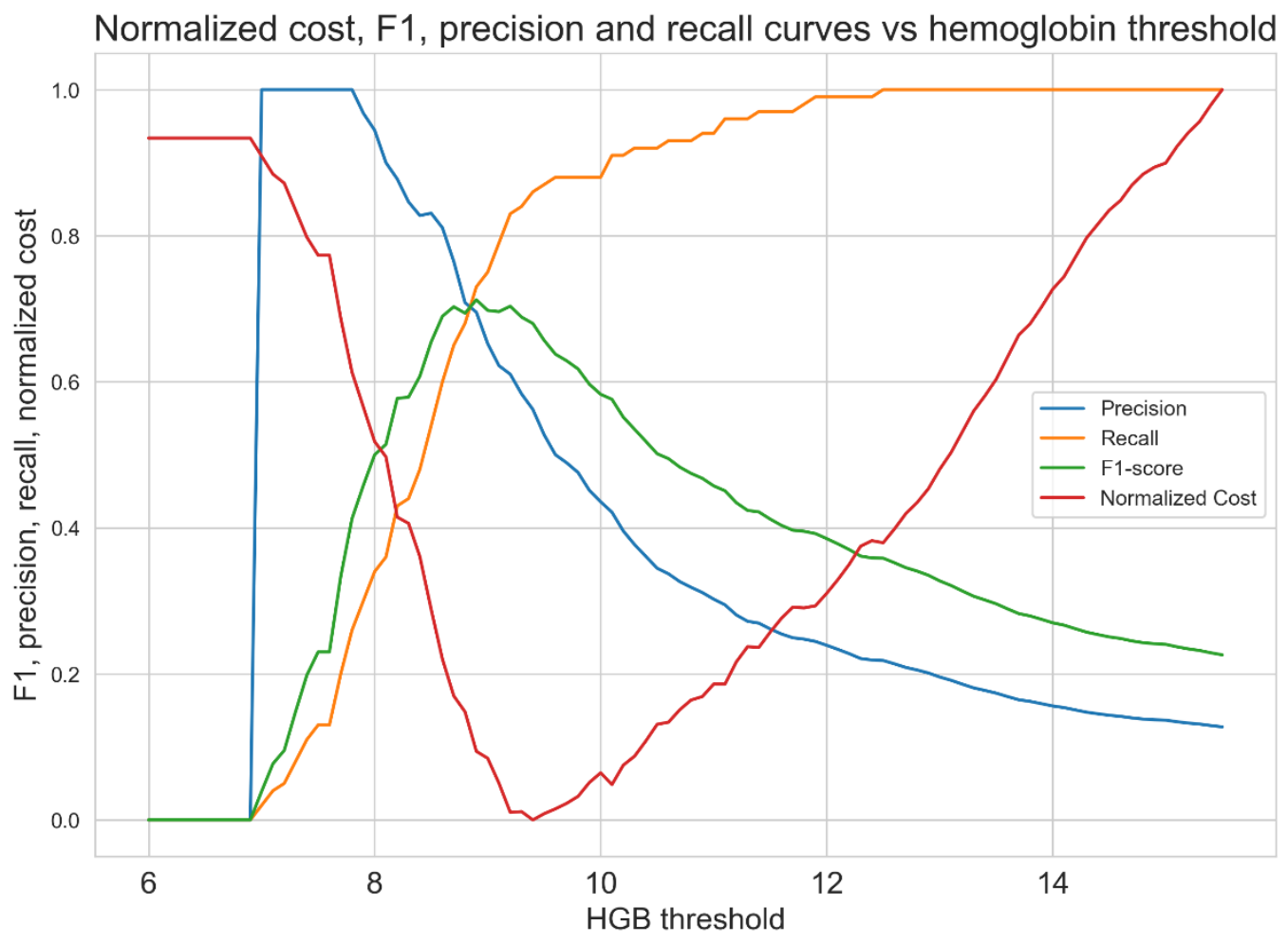

Figure 4. Metrics and costs variation in comparison to the changing HGB threshold. It is shown that the threshold with the lowest cost (red line) is $9.4 \mathrm{~g} / \mathrm{dL} \mathrm{HGB}$

\subsection{Evaluation of Costs from Machine Learning Models}

Table 2 shows the machine learning classification models performances, in form of $95 \%$ confidence interval for each computed metric. The best overall performing model is the Random Forest: in terms of both F1 score and costs. The Support Vector machine also achieves high AUC score; however, our choice is still the Random Forest. 
Table 2. Models performances. Lower and upper bounders of 95\% confidence interval are shown. CIs are computed on 10 -folds results

\begin{tabular}{llllll}
\hline & Precision & Recall score & F1-score & AUC & $\begin{array}{l}\text { Cost per patient } \\
\text { (average) }\end{array}$ \\
\hline Decision Tree & {$[0.68,0.85]$} & {$[0.58,0.72]$} & {$[0.57,0.70]$} & {$[0.91,0.93]$} & 29.81 euros \\
K-nearest neighbor & {$[0.70,0.78]$} & {$[0.59,0.66]$} & {$[0.63,0.7]$} & {$[0.88,0.92]$} & 25.61 euros \\
Logistic regression & {$[\mathbf{0 . 8 7}, \mathbf{0 . 9 7}]$} & {$[0.57,0.62]$} & {$[0.66,0.71]$} & {$[0.92,0.95]$} & 21.76 euros \\
Random Forest & {$[0.75,0.84]$} & {$[\mathbf{0 . 6 4 , 0 . 7 ]}$} & {$[\mathbf{0 . 6 9 , 0 . 7 6 ]}$} & {$[\mathbf{0 . 9 3 , 0 . 9 5 ]}$} & $\mathbf{1 8 . 2 6}$ euros \\
SVM & {$[0.78,0.86]$} & {$[0.60,0.65]$} & {$[0.68,0.72]$} & {$[\mathbf{0 . 9 3 , 0 . 9 5}]$} & 20.77 euros \\
Baseline method & {$[0.17,0.21]$} & {$[0.95,0.99]$} & {$[0.29,0.35]$} & {$[0.70,0.72]$} & 46.32 euros \\
Proposed method & {$[0.51,0.61]$} & {$[0.81,0.87]$} & {$[0.63,0.71]$} & {$[0.86,0.9]$} & $\mathbf{1 8 . 4 3}$ euros \\
& & & & & \\
\hline
\end{tabular}

The best performing model, i.e. the Random Forest, has also been trained with the unbalanced dataset (without applying SMOTE) to test the effectiveness of the rebalancing method. The nested-CV showed the following 95\% confidence intervals: $[0.7,0.82]$ for precision, $[0.63,0.68]$ for recall, $[0.62,0.75]$ for fl-score and $[0.92,0.94]$ for $A U C$. The rebalancing method led to slightly higher performances even if there are no statistically significant differences.

A graphic overview of the models results coming from the nested-CV, presented by table 2 , is given in figure 5, that shows the distribution of each performance measure over ten folds. Since nested-CV also performs the hyperparameters search, the models are tested with their best hyperparameters.
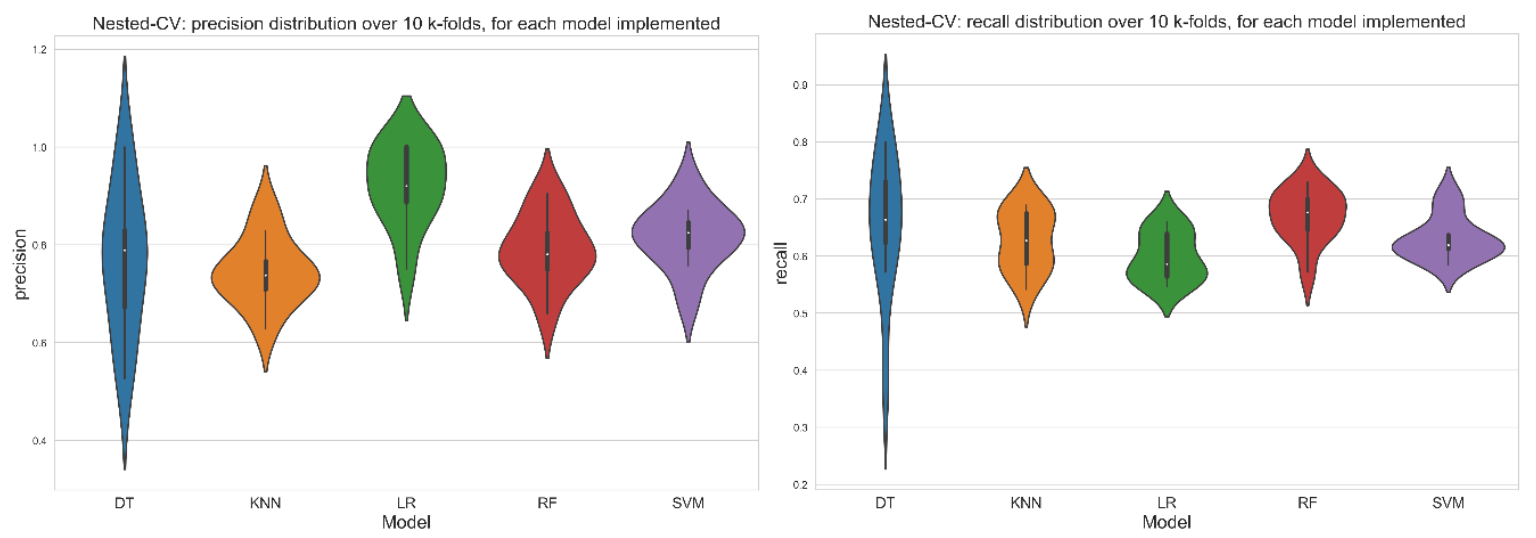

Nested-CV: f1-score distribution over $10 \mathrm{k}$-folds, for each model implemented
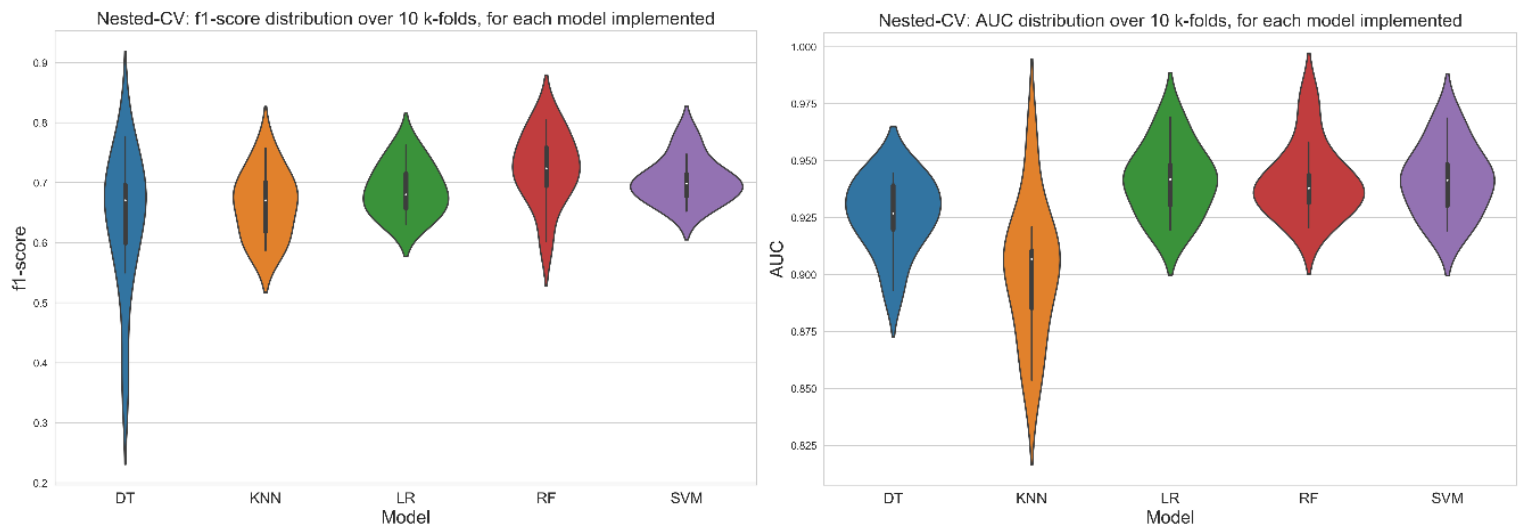

Figure 5. Violin plots representing the distribution of model performances over ten folds 


\section{DISCUSSION AND CONCLUSION}

Our methods show improvement in terms of cost savings in our setting.

In particular, the sensitivity analysis method allowed us to choose an optimal threshold, that is, a data driven decision rather than one that comes from experience. Clearly, this threshold must be validated in terms of clinical expertise, and we would like to validate our results in the future considering the medical knowledge of IOG's experienced staff. The threshold method can be interpreted in an intuitive way and it can also be used in an "offline setting".

On the other hand, the machine learning techniques are more of a black box model approach, so they cannot be used by just remembering one simple threshold. Instead, they require a computer interface that considers a multitude of variables. This second approach is different from the former and requires the use of an electronical device (such as, a computer or a tablet) for entry point to the models. However, the cost savings of a more complex model are higher than the simpler methods, and as such, one should think about whether the explainability and simplicity are more important to them rather than the increased cost savings.

Overall, we proposed two different methodologies for decreasing the costs of patient blood management. We explained each method's advantages and weaknesses, and both could lead to approximately $25 €$ of savings per patient (roughly $54 \%$ of initial cost per patient).

We would like to discover in the future which one is more appealing in a real-world setting, and our future work will build upon this foundation to propose a method that is applicable to real medical cases and to assess its performance and whether there is a real demand for it from the clinical staff or not.

\section{ACKNOWLEDGEMENT}

The authors are grateful to Alberto Cappelli from Healthcare Control Systems, Inc. (HCS), and to Francesco Magro, Chief Operating Officer of the IOG, for providing the dataset and useful information to conduct this research.

\section{REFERENCES}

Altman NS, 1992 "An introduction to kernel and nearest-neighbor nonparametric regression." The American Statistician 46(3):175-185

Chawla, Nitesh V., et al., 2002. "SMOTE: synthetic minority over-sampling technique." Journal of artificial intelligence research 16: 321-357.

Hayn, Dieter, et al., 2017. "Development of multivariable models to predict and benchmark transfusion in elective surgery supporting patient blood management." Applied clinical informatics 8.02 (2017): 617-631.

Hosmer Jr DW, Lemeshow S, Sturdivant RX (2013) “Applied logistic regression”, vol 398. John Wiley \& Sons

Kam HT, 1995. "Random Decision Forests.” In: Proceedings of the 3rd International Conference on Document Analysis and Recognition, Montreal, Canada, August, vol 1416, p 278282

Ramaravind K. Mothilal, Amit Sharma, and Chenhao Tan., 2020. "Explaining machine learning classifiers through diverse counterfactual explanations". In Proceedings of the 2020 Conference on Fairness, Accountability, and Transparency: 607-617

Sadana, Divyajot, et al., 2000. "Promoting high-value practice by reducing unnecessary transfusions with a patient blood management program." JAMA internal medicine 178.1: 116-122.

Safavian SR, Landgrebe D, 1991. "A survey of decision tree classifier methodology". IEE transactions on system, man, and cybernetics 21(3):660-674

Schölkopf B, Smola AJ, Bach F, et al., 2002. "Learning with kernels: support vector machines, regularization, optimization and beyond." MIT press

Zhang, S., 2012. Nearest neighbor selection for iteratively kNN imputation. Journal of Systems and Software, 85(11), 2541-2552. 